\title{
Dual Peroxidase and Colloidal Gold-Labeling Study of Angiotensin Converting Enzyme and Angiotensin-like Immunoreactivity in the Rat Subfornical Organ
}

\author{
Virginia M. Pickel, ${ }^{*}$ June Chan, ${ }^{\star}$ and Detlev Ganten $\dagger$ \\ *Laboratory of Neurobiology, Department of Neurology, Cornell University Medical College, New York, New York \\ 10021, and †German Institute of High Blood Pressure Research, University of Heidelberg, Heidelberg, Federal \\ Republic of Germany
}

\begin{abstract}
The cellular relationships between angiotensin converting enzyme (ACE) (EC 3.4.14.1) and angiotensin-like immunoreactivity (AGLI) were examined in the subfornical organ (SFO). Brains from adult rats were fixed by vascular perfusion with $3.75 \%$ acrolein and $2 \%$ paraformaldehyde. The region containing the SFO was then sectioned on a vibrating microtome. Partially permeabilized sections were immunocytochemically labeled using the peroxidase-antiperoxidase (PAP) or combined PAP and immunogold methods. Goat antiserum to ACE was localized to both non-neuronal and neuronal cells within the SFO. Intense peroxidase immunoreactivity for $A C E$ was associated with the ventricular and basal surface of ependymal cells, the luminal surface of the vascular endothelium, portions of glial membranes exposed to extracellular spaces, and membranous organelles within neuronal processes.

Two antisera raised in rabbits against angiotensin II showed peroxidase immunoreactivity within the extracellular spaces and throughout the cytoplasm of numerous axon terminals and a few perikarya and dendrites in the SFO. Axon terminals and dendrites also showed aggregates of AGLI in smooth membranes and vesicles near the plasmalemma. Gold labeling for AGLI was evident in only $6 \%$ of the axon terminals and in a smaller number of dendrites containing peroxidase immunoreactivity for $\mathrm{ACE}$. The low incidence of terminals containing both markers appeared to at least partially reflect limited penetration of the $10 \mathrm{~nm}$ gold particles. These results provide the first ultrastructural evidence that $\mathrm{ACE}$ is associated with the plasmalemma and membranous organelles strategically located for interaction with precursors of angiotensin II or other peptides within the cerebrospinal fluid, extracellular spaces and neurons of the SFO.
\end{abstract}

Angiotensin II ( $\mathrm{Ag} \mathrm{II)}$ is produced by the removal of 2 carboxyterminal amino acids from angiotensin $\mathrm{I}(\mathrm{Ag} \mathrm{I})$ through the action of angiotensin converting enzyme (ACE, EC 3.4.14.1) (Soffer, 1976). The enzyme is principally found along the luminal surface of capillary endothelium in the lung and other peripheral organs (Das and Soffer, 1976; Ryan et al., 1975). However, there is increasing evidence that ACE may be involved in the neuronal synthesis of $\mathrm{Ag}$ II in the CNS (Ganten et al., 1983). The findings are somewhat controversial in that

Received Nov. 6, 1985; revised Feb. 11, 1986; accepted Feb. 17, 1986.

This research was supported by NIH Grant HL 18974 and by a career award from NIMH (MH 00078). The aulhors are also indebted to Dr. R. L. Soffer for supplying the antiserum to angiotensin converting enzyme.

Correspondence should be addressed to Dr. Virginia M. Pickel, Laboratory of Neurobiology, Department of Ncurology, Cornell University Medical College, 411 East 69th Street, New York, NY 10021.

Copyright (C) 1986 Society for Neuroscience $0270-6474 / 86 / 082457-13 \$ 02.00 / 0$
Ag II antisera from only a few laboratories can be immunocytochemically localized to neuronal pathways in brain (Brownfield et al., 1982). Furthermore, many of the pathways with angiotensin-like immunoreactivity (AGLI) do not contain immunocytochemically detectable levels of ACE (Brownfield et al., 1982). Possible explanations for these discrepancies may include cross-reaction of the Ag II antisera with closely related peptides; use of immunocytochemical labeling methods that fail to detect antigenic sites within the plasmalemma or other membranous organelles; and reliance on single markers and lightmicroscopic immunocytochemistry. To evaluate these possibilities, we examined the ultrastructural localization of ACE and Ag II in the rat subfornical organ (SFO) using immunoblots to characterize 2 (CE and DE) antisera against the peptide and a variety of fixation- and penetration-enhancing methods to ensure optimal labeling with 2 immunocytochemical markers. The SFO was chosen because of its close anatomical and functional relationships with both the peripheral and central angiotensin systems (Lind et al., 1983; Simpson, 1981). Furthermore, the SFO is known to contain detectable levels of AGLI and ACE as seen by light-microscopic immunocy tochemistry (Brownfield et al., 1982; Lind et al., 1984). We sought to determine (1) what was the ultrastructural localization of ACE with respect to ependymal, vascular, and neuronal components of the SFO, (2) how the ultrastructural localization of immunoreactive $\mathrm{Ag}$ II compared to ACE, and (3) whether ACE and AGLI could be dually localized by combined peroxidase-antiperoxidase (PAP) (Sternberger, 1974) and immunogold (De Mey et al., 1982) methods.

\section{Materials and Methods}

\section{Animals}

Twenty-five male Sprague-Dawley rats, 200-300 gm (Hilltop Lab Animals, Inc.), were used in the study. All of the animals were anesthetized with Nembutol $(50 \mathrm{mg} / \mathrm{kg}$, i.p.) and perfused through the ascending aorta with various fixatives.

\section{Fixation- and penetration-enhancing methods}

The fixation conditions required to give optimal labeling of antisera for $\mathrm{ACE}$ and Ag II were experimentally determined. Aldehyde fixatives were preceded by vascular perfusion with $10 \mathrm{ml}$ of $0.9 \% \mathrm{NaCl}$ containing $1000 \mathrm{U} / \mathrm{ml}$ of heparin. The first group of 6 rats was then perfused for $10 \mathrm{~min}$ with $200 \mathrm{ml}$ of $0.2 \%$ glutaraldehyde and $4 \%$ paraformaldehyde in $0.1 \mathrm{M}$ phosphate buffer, $\mathrm{pH} 7.4$. The brains were removed and subsequently placed in the same fixative for an additional $45 \mathrm{~min}$. Microscopic examination revealed better preservation of ultrastructural morphology and immunocytochemical labeling using acrolein rather than glutaraldehyde. The procedure consisted of a $2 \mathrm{~min}$ perfusion with 75 $\mathrm{ml}$ of $3.75 \%$ acrolein (Polysciences) and $2 \%$ paraformaldehyde in 0.1 $\mathrm{M}$ phosphate buffer, $\mathrm{pH} 7.4$, followed by continued perfusion with 200 $\mathrm{ml}$ of $2 \%$ paraformaldehyde and immersion fixation for $30 \mathrm{~min}$ in the 

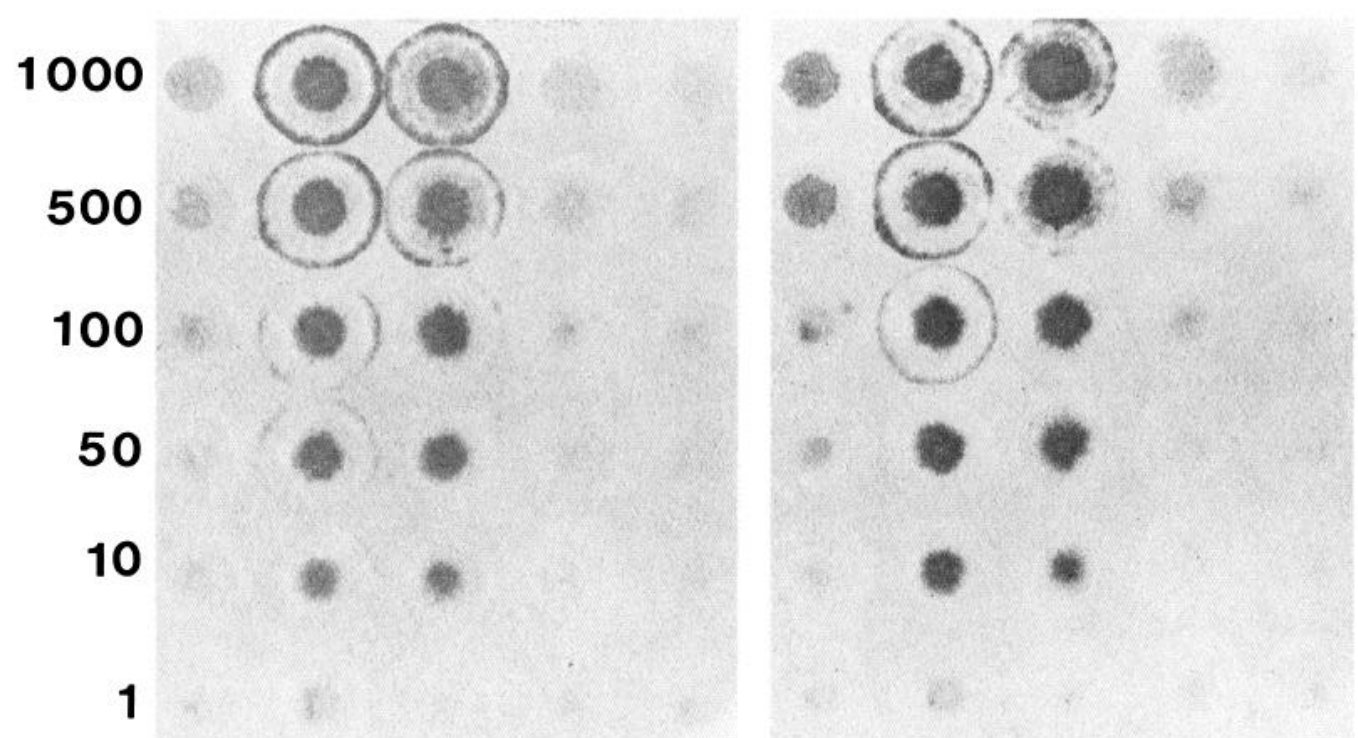

\section{Ag-I Ag-II Ag-III RS VAS Ag-I Ag-II Ag-III RS VAS}

Figure 1. Dot-blots showing the cross-reaction of $2 \mathrm{Ag} \mathrm{II}$ antisera, CE (left) and DE (right) with 1-1000 ng quantities of angiotensin I ( $A g$ - $I$ ), angiotensin II $(A g-I I)$, angiotensin III $(A g-I I I)$, renin substrate $(R S)$, and vasopressin $(V A S)$. Both CE and DE antisera were used at a $1: 1000$ dilution.

paraformaldehyde solution. This latter perfusion method was used for the remaining animals.

With both fixation procedures, peroxidase immunoreactivity for ACE was virtually nondetectable without further treatment to enhance penetration of the antisera. The penetration-enhancement methods included (1) rapid freeze-thawing of tissues cryoprotected with $30 \%$ sucrose, (2) two min incubations of vibratome sections through an ascending and descending graded ethanol series extending to $40 \%$ ethyl alcohol in phosphate buffer, or (3) inclusion of $0.01 \%$ saponin in the primary antiserum. The alcohol treatment was generally found to give the best preservation of ultrastructural morphology, though the intensity of the immunocytochemical labeling was greater with saponin.

\section{Antisera}

Details for purification of ACE from rabbit lung, production of a polyclonal antiserum in goats, and criteria of specificity of the antiserum have been described previously (Das and Soffer, 1976). The ACE-antiserum was produced and generously supplied by Dr. R. L. Soffer, Cornell University Medical College. The CE and DE antisera were produced in rabbits following injections of 5-Ile-Ag II previously conjugated to BSA by means of glutaraldehyde. The $\mathrm{CE}$ and $\mathrm{DE}$ antisera against $\mathrm{Ag}$ II have been tested for cross-reactivity with renin substrate (RS) and Ag I, II, and III using radioimmunoassay (Brownfield et al., 1982).

\section{Single labeling with the PAP method}

The procedure for immunocytochemical labeling by the PAP method has been described in detail (Pickel, 1981; Sternberger, 1974). Briefly, coronal $(30 \mu \mathrm{m})$ Vibratome sections were collected throughout the rostrocaudal extent of each SFO. These sections were incubated with 1:1000 dilutions of primary antisera for 18-24 hr at room temperature. The goat antibody to ACE was then followed by $30 \mathrm{~min}$ incubations in a 1:50 dilution of rabbit anti-goat immunoglobulin $(\mathrm{IgG})$ and a 1:100 dilution of goat PAP. Incubations with rabbit antisera to $\mathrm{Ag}$ II were followed by goat anti-rabbit IgG and rabbit PAP using the same incubation periods and dilutions of respective sera. The dilutions and washes between incubations were in $0.1 \mathrm{M}$ Tris-saline, $\mathrm{pH}$ 7.4. The peroxidase was finally reacted with 3,3'-diaminobenzidine and hydrogen peroxide.

\section{Dual labeling with PAP and colloidal gold}

Additional sections from the same animals were processed for the dual localization of ACE and angiotensin using the PAP and immunogold methods. At the end of the first PAP-labeling procedure for ACE, the sections were washed in Tris-saline $(3 \times 10 \mathrm{~min})$, then incubated for
$24 \mathrm{hr}$ with a 1:1000 dilution of the $\mathrm{CE}$ antiserum to $\mathrm{Ag}$ II followed by $2 \times 15$ min washes in Tris-saline, $2 \times 5$ min washes in $1 \%$ BSA in Trissaline, and a $2 \mathrm{hr}$ incubation in a 1:10 dilution of goat anti-rabbit IgG conjugated to $10 \mathrm{~nm}$ gold particles (Structure Probe, West Chester, PA). Following incubation with the gold-IgG, the sections were washed $3 \times$ $5 \mathrm{~min}$ in Tris-saline and placed in $1 \%$ glutaraldehyde in phosphate buffer for $10 \mathrm{~min}$ (De Mey et al., 1982). Omission of the first or second antiserum or substitution of adsorbed control sera revealed the absence of nonspecific interactions when the PAP labeling preceded the immunogold series. However, when the procedure was reversed, randomly scattered gold particles and reduced specific labeling in neuronal structures were frequently observed.

\section{Light- and electron-microscopic preparations and data analysis}

Single- and dual-labeled sections were subsequently processed for light or electron microscopy. The light-microscopic sections were mounted on gelatin-coated slides, coverslipped, and examined with bright-field or Nomarski optics. For electron microscopy, the above labeling procedure was followed by postfixation for $1 \mathrm{hr}$ in $2 \%$ osmium tetroxide in phosphate buffer. The sections were then dehydrated and flat-embedded in Epon 812. Ultrathin sections were collected from the outer surface of the tissue and examined with a Philips 201 EM.

\section{Controls}

The CE and DE antisera against $\mathrm{Ag}$ II were tested for cross-reactivity by 2 methods. In the first, the specific anti-IgGs were adsorbed using commercially bought peptides. These included $\mathrm{Ag} \mathrm{I}$, II, and III, RS, vasopressin, and bradykinin (Peninsula Labs.). Each peptide, $50 \mu \mathrm{g}$, was added to $1 \mathrm{ml}$ of the primary antiserum at the working dilution of 1:1000. After $2 \mathrm{hr}$ of incubation at $37^{\circ} \mathrm{C}$, the complexes were removed by centrifugation, and the supernatant was substituted for the primary antiserum in the immunocytochemical labeling procedure.

The second method used immunoblots in which the commercially bought peptides were dissolved in water to yield a concentration of 1 $\mathrm{mg} / \mathrm{ml}$. These were then serially diluted and dropped in wells on strips of Whatman No. 1 filter paper held by a filtration manifold (Schleicher and Schuell). Each well in the filtration unit contained $10 \mu \mathrm{l}$ of the peptide at concentrations ranging from 1 to $1000 \mathrm{ng}$. The peptides were air-dried on the paper and exposed to paraformaldehyde vapor at $80^{\circ} \mathrm{C}$ for $1 \mathrm{hr}$. These papers were then stained immunocytochemically with a 1:1000 dilution of the $\mathrm{CE}$ and $\mathrm{DE}$ antisera to $\mathrm{Ag} \mathrm{II}$ according to the procedure of Larsson (1981). 

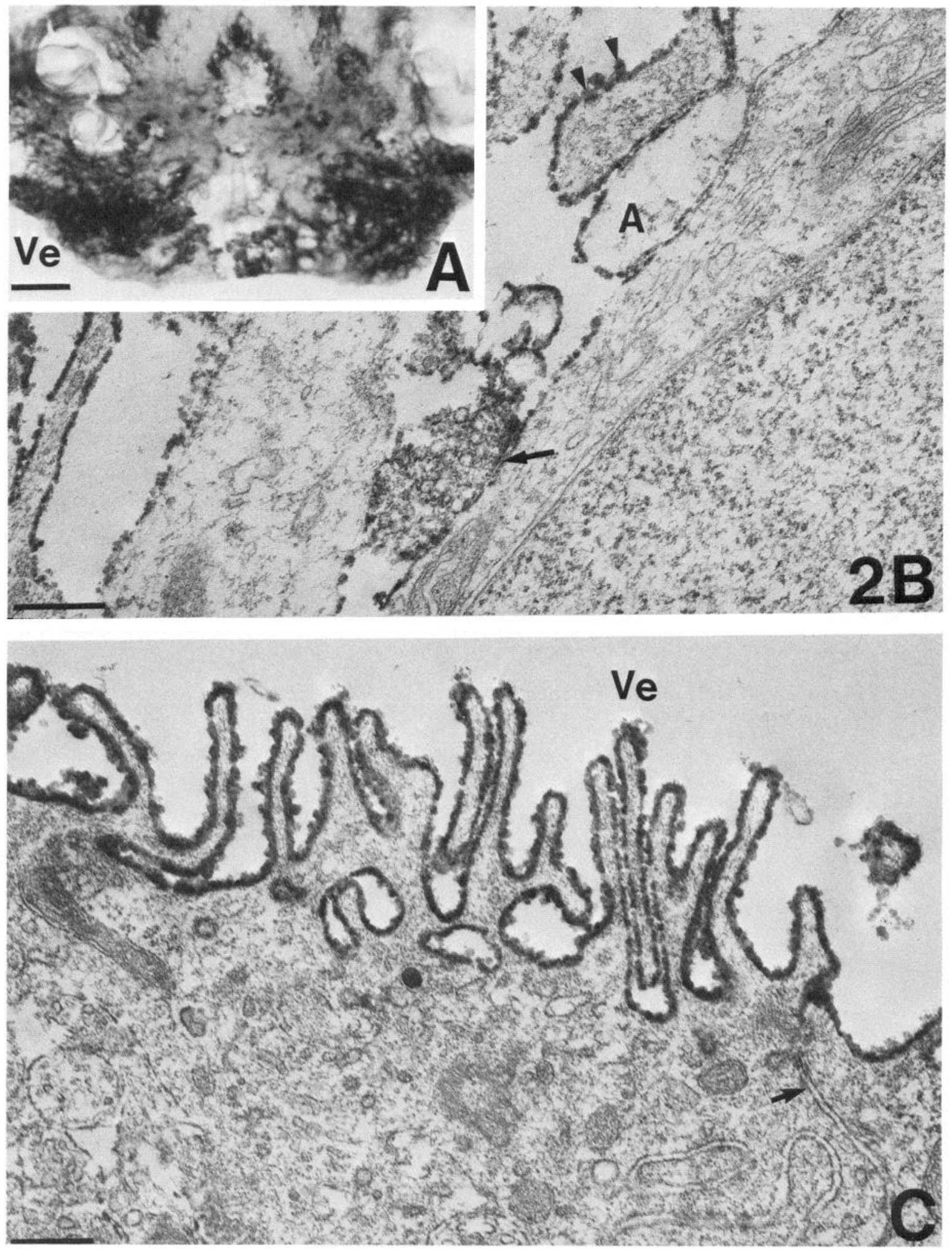

Figure 2. Localization of ACE in SFO. A, Light micrograph shows diffuse labeling of ACE near the ventricular $(V e)$ surface and throughout the interstitial regions in the ventrolateral portions of the SFO. Bar, $50 \mu \mathrm{m}$. B, Electron micrograph shows the punctate localization (arrowheads) of immunoreactivity of ACE along the plasma membranes of the basal processes of ependymal cells. Peroxidase reaction for ACE also rims small vesicles in a process contacting the plasmalemma of an unlabeled cell at the arrow. Electron-lucent process presumed to be astrocytic $(A)$ also shows immunoreactivity along the plasmalemma. Bar, $0.6 \mu \mathrm{m}$. $C$, Electron micrograph shows the granular localization of immunoreactivity for ACE along the microvillar surface of an ependymal cell in direct contact with the ventricle ( $V e$ ). Peroxidase reaction is not seen at the junction (arrow) between adjacent ependymal cells. Bar, $0.3 \mu \mathrm{m}$. 


\section{Results}

Cross-reactivity of the antisera

In the dot-blots, both the CE and the DE antisera showed slightly more cross-reactivity with $\mathrm{Ag}$ II than with $\mathrm{Ag} I$ or III using small (1-10 ng) quantities of the peptide (Fig. 1). However, at higher concentrations, both antisera showed approximately equal cross-reaction with $\mathrm{Ag}$ II and $\mathrm{Ag}$ III. The cross-reactions with $\mathrm{Ag}$ I and RS were slightly greater for the DE than the CE. There was no detectable cross-reaction with vasopressin (Fig. 1) or with bradykinin (not shown).

In adsorption control studies, the immunocytochemical labeling was not detectable in sections incubated with $\mathrm{CE}$ or $\mathrm{DE}$ antiserum previously combined with $50 \mu \mathrm{g}$ quantities of $\mathrm{Ag} \mathrm{II}$ or III. Adsorption with $50 \mu \mathrm{g} \mathrm{Ag} \mathrm{I}$ also appeared to reduce slightly the detectable immunoreactivity with both the CE and $\mathrm{DE}$ antisera. Unless otherwise indicated, the results of the present study were obtained with the CE antiserum. Adsorption of ACE with a partially purified homogenate of the enzyme also removed all detectable immunoreactivity.

\section{Non-neuronal localization of $A C E$ and $A G L I$}

By light microscopy, peroxidase immunoreactivity for $\mathrm{ACE}$ was intensely localized along the ventricular surface, the basolateral regions near the choroid plexus, and around sinusoidal and other blood vesscls in the SFO (Fig. 2A). Electron-microscopic examination revealed ACE-like immunoreactivity principally associated with the plasmalemma of ependyma and astrocytes. The labeling was especially intense along the basal (Fig. $2 B$ ) and apical (Fig. 2C) surfaces of the ependymal cells. ACE-like immunoreactivity was absent at the junctions between neighboring ependymal cells.

The peroxidase immunoreactivity for ACE was also especially dense along the luminal surface of endothelial cells (Fig. 3A) and basal surfaces of perivascular astrocytes (Fig. 3). The processes of single or neighboring astrocytes often enclosed small groups of unlabeled neuronal processes and surrounding extracellular spaces (Fig. 3B). Comparable extracellular spaces near blood vessels frequently showed diffuse and intense AGLI (Fig. $4, A, B)$.

\section{Neuronal localization of $A C E$ and $A G L I$}

The principal neuronal localization of AGLI was in varicose axon terminals located toward the more central regions of the SFO (Figs. 4, $A, C ; 5$ ). Most of the labeled terminals contained numerous large $(80-100 \mathrm{~nm})$ granular or agranular vesicles that were heavily rimmed with peroxidase immunoreactivity and located distal to the synaptic specialization (Fig. 4C). They also contained a few smaller $(40-60 \mathrm{~nm})$ clear vesicles that were aggregated near the synaptic junctions. The synapses were primarily symmetric contacts with unlabeled dendritic shafts. A second type of labeled terminal contained 0-2 large dense vesicles and numerous smaller vesicles evenly distributed throughout the cytoplasm of terminals examined in single sections (Fig. $5, A, B)$. The latter group of terminals formed symmetric synapses with unlabeled perikarya and dendrites, and contained diffuse AGLI rimming all membranous organelles. Many of the recipient dendrites showed pinocytotic vesicles at points contacting extracellular spaces. Frequently the extracellular spaces near the terminals with AGLI also contained small $(0.1-0.2 \mu \mathrm{m})$ unlabeled neuronal processes (Fig. 5). These spaces were delineated by glial processes and sometimes also contained AGLI. A third group of axon terminals showed dense accumulations of AGLI in saccules of membranes and vesicles near the plasmalemma at sites distal to the synaptic junctions (Fig. 6 $\mathrm{A}$ ).

The peroxidase labeling for ACE within axon terminals paralleled that of $\mathrm{Ag}$ II but differed in terms of intensity and subcellular localization of the reaction product. A few small (0.1-
$0.5 \mu \mathrm{m})$ processes near the basal surfaces of ependymal cells contained dense peroxidase immunoreactivity around small (40$60 \mathrm{~nm}$ ) clear vesicles (Fig. $2 B$ ). However, these processes were infrequently detected, and their size and density suggested that they might be surface sections through ependymal rather than neuronal processes. The majority of the unequivocal axon terminals showed extremely faint labeling for ACE associated with synaptic vesicles. Heavier accumulations of immunoreactivity were detected in a few membranes and vesicles near the plasmalemma. These membranes and associated vesicles sometimes appeared to be in continuity with the plasmalemma of preterminal axons (Fig. $6 B$ ). In dual studies, colloidal gold marker for AGLI was detected in a few (15 out of 250 , or $6.0 \%$ ) of the terminals showing peroxidase labeling for ACE (Fig. 6C). Comparison of the number of terminals labeled for $\mathrm{Ag}$ II with the immunogold versus PAP methods revealed that many fewer terminals were labeled by gold than peroxidase. The superficial location of the gold particles indicated that poor penetration largely accounted for the small number of terminals labeled by the immunogold method. Terminals labeled with gold were arbitrarily designatcd as those containing at lcast 10 times the number of particles seen in a comparable area of surrounding tissue.

Perikarya containing peroxidase AGLI were few in number and most evident near the ventricular surface of sections through the SFO that were labeled with the DE antiserum. By light microscopy, dense reaction product was seen throughout the cytoplasm of selective perikarya and their proximal processes (Fig. 7, $A, B$, insert). In favorable planes of section, a labeled process sometimes could be followed toward the ventricular surface but could not be traced as far as the ependymal cells. Perikarya with AGLI contained a round, unindented nucleus with peripheral clumps of heterochromatin. These perikarya received axosomatic synapses and exhibited numerous saccules of smooth reticulum in a relatively thin rim of cytoplasm (Figs. $7 B ; 8 A$ ). The labeled cells usually were separated from the ependymal layer by one or more unlabeled perikarya and processes (Fig. 7B).

Most dendrites with AGLI were located near the ventricular surface and received synapses from unlabeled axon terminals. The labeled dendrites usually contained diffuse peroxidase reaction throughout the cytoplasm (Fig. $8 B$ ). However, heavier accumulations of immunoreactivity were seen in association with saccules of smooth reticulum and vesicles near the plasmalemma (Figs. $6 A ; 8 B$ ). In dual-labeling studies, a few of the dendrites contained immunoreactivity for ACE and Ag II (Fig. $8 C$ ). The peroxidase labeling for ACE appeared especially dense along membranous structures in portions of the dendrites apposed to extracellular spaces (Fig. $8 \mathrm{C}$ ). These spaces were delineated by glial processes and also contained small unlabeled neuronal processes. The immunogold labeling for $\mathrm{Ag}$ II was equally distributed throughout the dendritic cytoplasm. As described for the gold labeling in axon terminals, relatively few dendrites showed significant (10 times background) accumulations of gold particles and were located extremely near the surface of the tissue.

\section{Discussion}

Our findings demonstrate that, in the adult rat SFO, peroxidase immunoreactivity for ACE is principally localized to the plasmalemma of ependymal cells and astrocytes in contact with the cerebrospinal fluid (CSF) and extracellular spaces near blood vessels, to the luminal surface of capillary endothelial cells, and to saccules of smooth reticulum and vesicles near the plasmalemma in a few axon terminals and dendrites. We have also shown that AGLI cross-reacts primarily with $\mathrm{Ag}$ II and III and is extensively localized in extracellular spaces near blood vessels and in axon terminals and a few perikarya and dendrites in the 

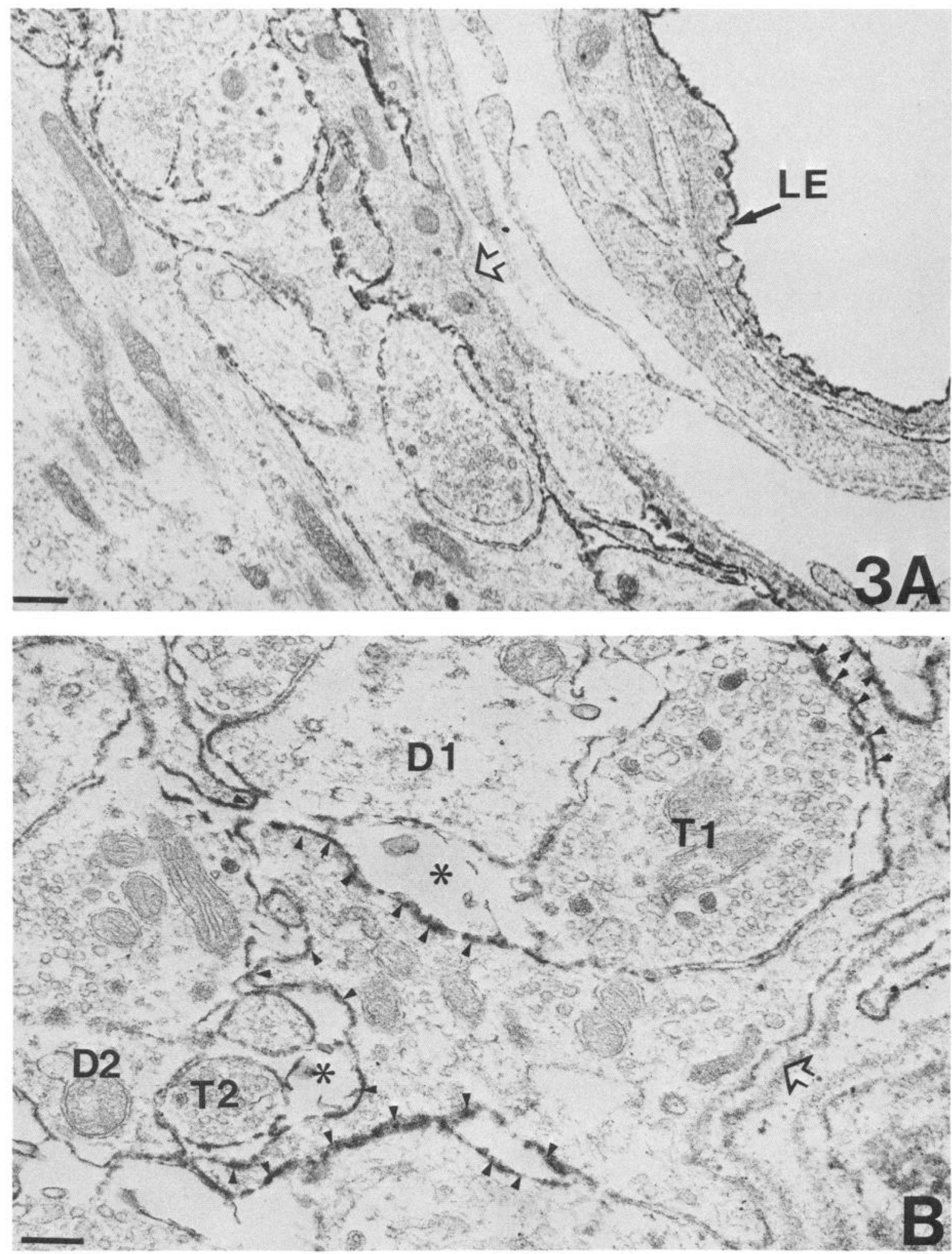

Figure 3. Ultrastructural localization of immunoreactive ACE in association with blood vessels of SFO. A, Low-magnification micrograph shows the localization of peroxidase immunoreactivity for the enzyme along the luminal surface of a vascular endothelial cell $(L E)$ and along the plasma membranes near the brush border (open arrowhead) outside the perivascular space. Bar, $0.5 \mu \mathrm{m}$. B, Higher-magnification electron micrograph of the perivascular region shows intense peroxidase immunoreactivity (small arrowheads) along the basal process of an astrocyte abutting the vascular brush border (open arrow). The labeled astrocytic processes surround unlabeled terminals ( $T 1$ and $T 2$ ), dendrites (D1 and D2), and extracellular spaces (asterisk). Bar, $0.3 \mu \mathrm{m}$. 

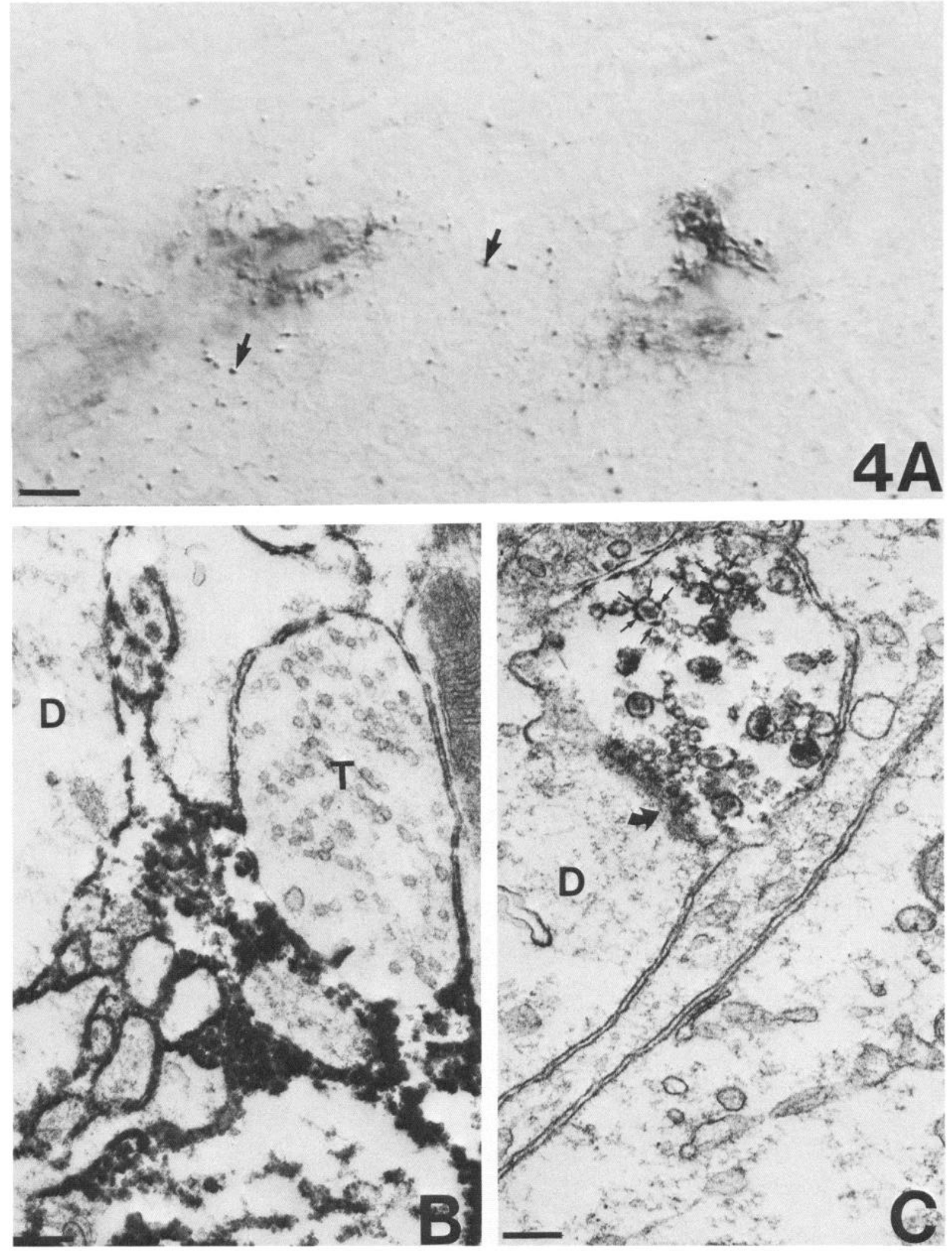

Figure 4. Extracellular and terminal localization of AGLI in the SFO. A, Light micrograph shows peroxidase immunoreactivity in diffuse patches around blood vessels and in punctate varicosities (arrows) in the central region of the SFO. Bar, $50 \mu \mathrm{m}$. B, Electron micrograph demonstrates the peroxidase reaction for AGLI between an unlabeled axon terminal $(T)$ and dendrite $(D)$, which are invested by glial processes. Bar, $0.2 \mu \mathrm{m}$. $C$, Electron micrograph shows the localization of AGLI in an axon terminal containing numerous large dense core and both small and large opaque vesicles. Some of the vesicles are heavily rimmed (small arrows) with peroxidase reaction. The labeled terminal forms a symmetric junction (curved arrow) with unlabeled dendrite $(D)$. Bar, $0.2 \mu \mathrm{m}$. 

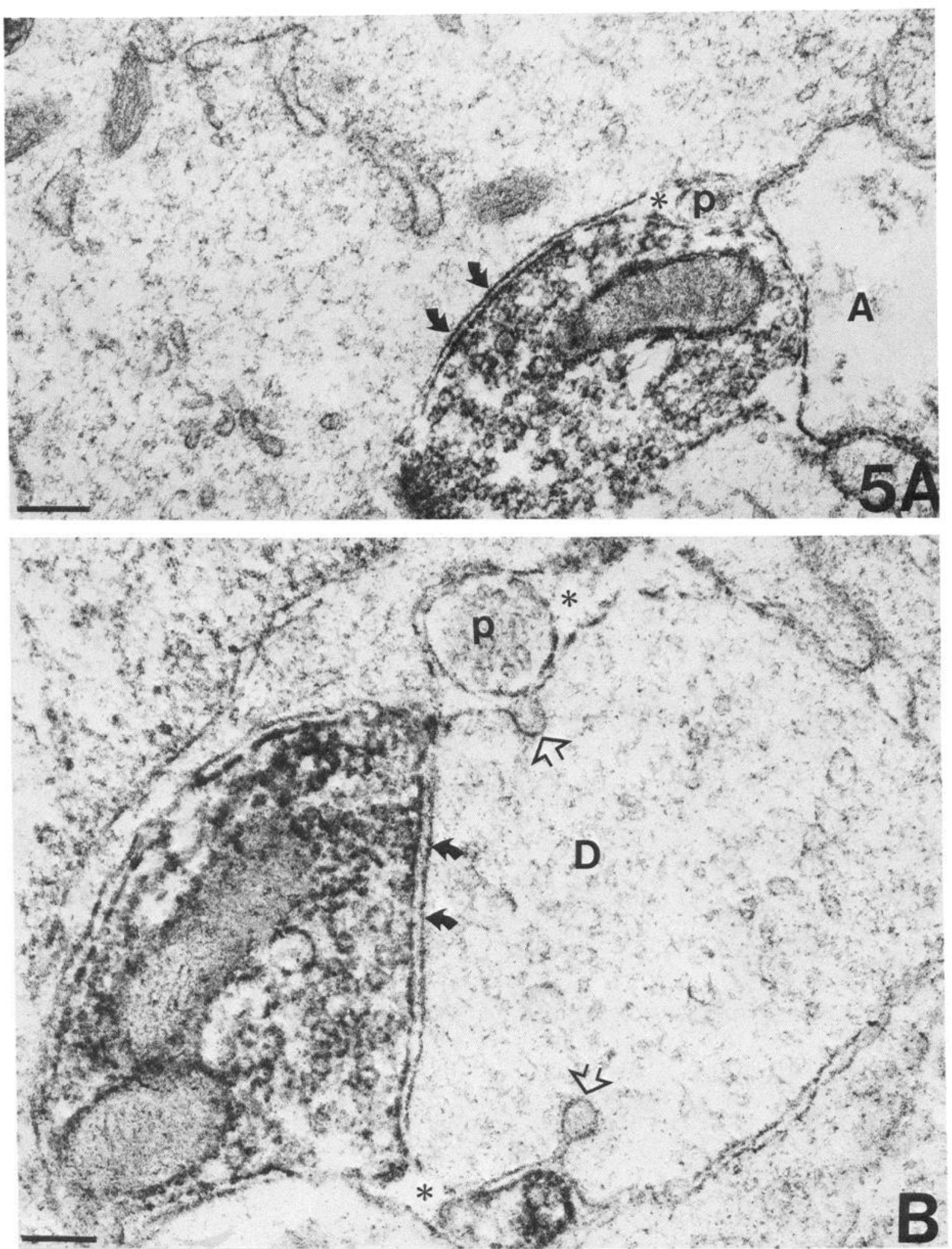

Figure 5. Axosomatic and axodendritic synapses formed by terminals with AGLI in the SFO. A, Electron micrograph shows the localization of AGLI in an axon terminal forming a symmetric synapse (curved arrows) with an unlabeled neuronal perikaryon. The labeled terminal contains primarily small clear vesicles. An astrocytic process $(A)$ also shows AGLI along the plasmalemma in contact with the extracellular space (asterisk). The astrocytic process appears to isolate the labeled terminal along with an unlabeled process $(p)$. Bar, $0.2 \mu \mathrm{m}$. $B$, Electron micrograph illustrates the localization of AGLI in an axon terminal forming a symmetric syapse (curved arrows) with an unlabeled dendrite $(D)$. Pinocytotic vesicles (open arrowheads) appose the extracellular spaces (asterisk) and both labeled and unlabeled processes (p). Bar, $0.2 \mu \mathrm{m}$. 

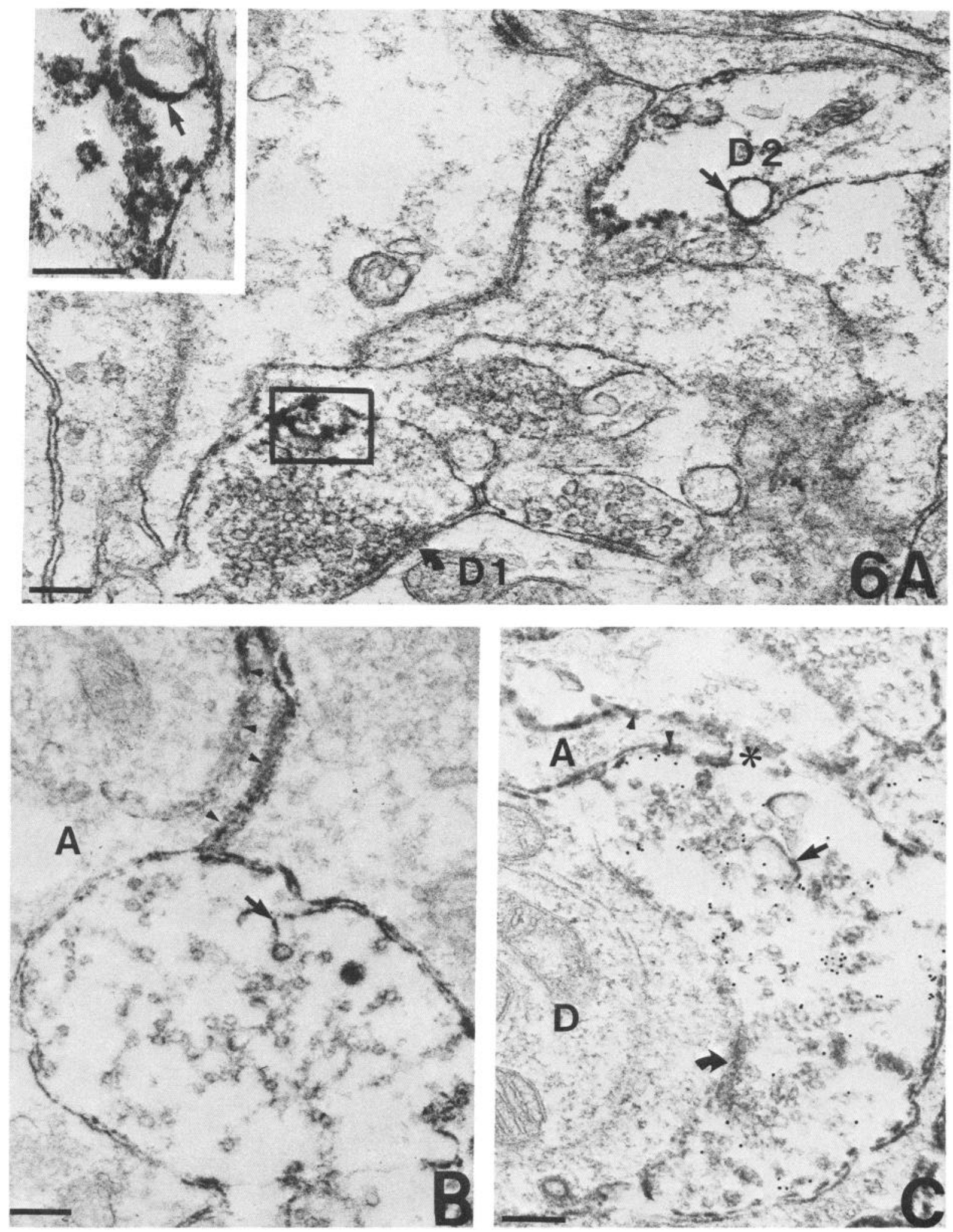

Figure 6. Localization of AGLI and ACE in neuronal processes in the SFO. A, Electron micrograph shows membrane-associated packet near the plasmalemma of an otherwise unlabeled axon terminal. The higher-magnification insert of the boxed region in the axon terminal illustrates dense immunoreactivity along a membranous structure (arrow) in direct continuity with the plasmalemma. Smaller vesicles in the immediate vicinity are also heavily rimmed with immunoreactivity. The labeled terminal forms a symmetric synapse (curved arrow) with an unlabeled dendrite (DI). A second dendrite $(D 2)$ shows accumulations of immunoreactivity along membranous saccules near the plasmalemma. Bar, $0.2 \mu \mathrm{m}$. $B$, Electron micrograph shows the localization of ACE in a preterminal axon. Dense immunoreactivity is associated with a membranous structure (arrow) near the plasmalemma and around numerous granular and agranular vesicles. Astrocytic process $(A)$ also shows immunoreactivity $($ arrowheads) along the plasmalemma. To facilitate detection of immunoreactivity, the section was not counterstained with lead citrate. Bar, $0.2 \mu \mathrm{m}$. $C$, Electron micrograph shows dual peroxidase labeling for ACE around saccules of reticulum (arrow), synaptic vesicles, and astrocytic $(A)$ process $($ arrowheads) and immunogold labeling for $\mathrm{Ag}$ II throughout the axon terminal. A junction (curved arrow) is seen with unlabeled dendrite $(D)$. Bar, $0.2 \mu \mathrm{m}$. 

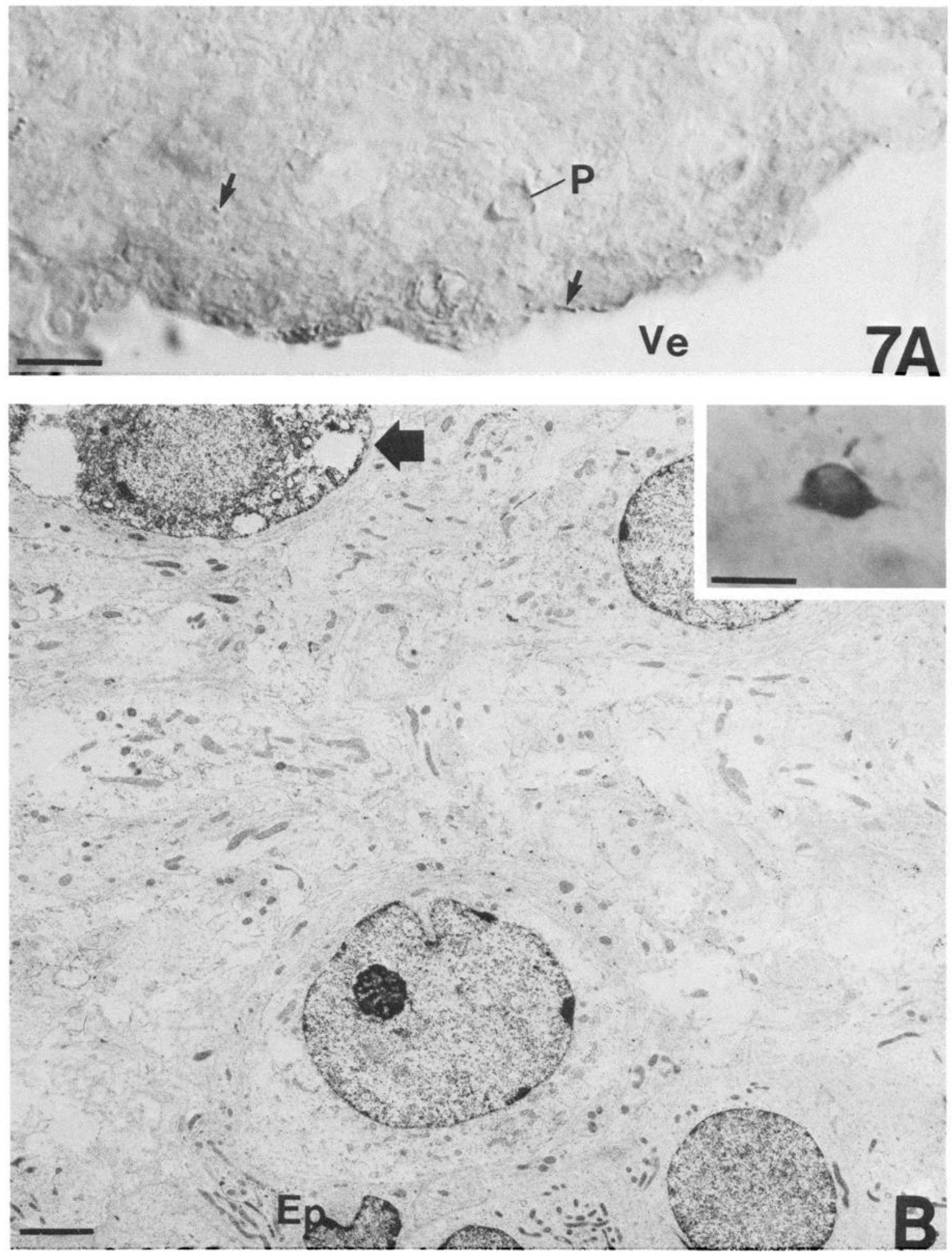

Figure 7. Localization of AGLI in perikarya in the SFO. A, Light micrograph shows the localization of peroxidase immunoreactivity in a perikaryon $(P)$ and several punctate processes (arrows) near the ventricular $(V e)$ surface. Bar, $50 \mu \mathrm{m}$. $B$, Electron micrograph shows the ultrastructural localization of AGLI in a similar perikaryon. Other unlabeled perikarya separate the immunoreactive soma (large arrow) from overlying ependymal cells (Ep). Light micrograph (insert at right) shows the same labeled cell photographed prior to sectioning for ultrastructural examination. Electron-dense nuclei were seen in both control and immunocytochemically labeled sections. Bar, $1.3 \mu \mathrm{m}$ in electron micrograph; $50 \mu \mathrm{m}$ in insert. 

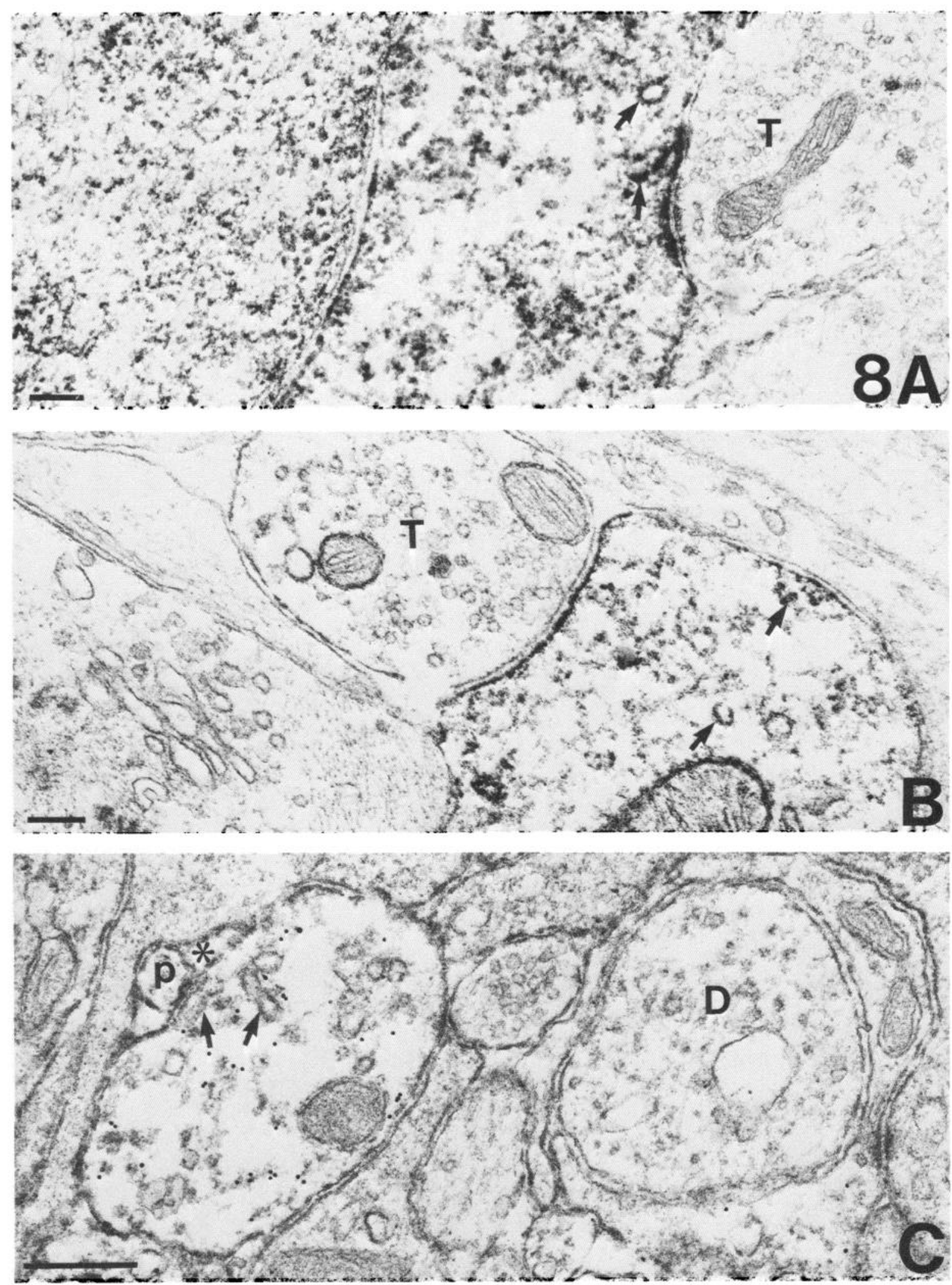

Figure 8. Perikaryal and dendritic localization of AGLI and ACE in the SFO. A, Electron micrograph shows AGLI in the cytoplasm of a neuronal perikaryon. Intense labeling is seen along membranous saccules and vesicles (arrow) near the somatic synapse with terminal $(T)$. $B$, Electron micrograph shows AGLI diffusely distributed in the cytoplasm with heavier accumulations of immunoreactivity along membranous saccules (arrows) of a dendrite postsynaptic to terminal (T). C, Electron micrograph shows ACE immununoreactivity as dense peroxidase product (arrows) along saccules of vesicles in contact with the extracellular space (asterisk) and unlabeled processes (p). The same dendrite contains AGLI, as indicated by immunogold labeling. An unlabeled dendrite $(D)$ is seen at right. Bar, $0.3 \mu \mathrm{m}$ throughout. 
SFO. Our dual labeling with peroxidase and gold has shown the coexistence of ACE and AGLI in the same neuronal processes in the SFO. These results are discussed in rclation to thcir anatomical and functional implications.

\section{Ultrastructural localization of $A C E$}

\section{Plasmalemma of ependyma and astrocytes}

The present localization of ACE along ependymal surfaces in the SFO supports previous light-microscopic immunocytochemical studies demonstrating reaction product for renin ( $\mathrm{Rix}$ et al., 1981) and ACE (Brownfield et al., 1982) in association with ependyma in rat brain. Brownfield et al. (1982) also noted diffuse, but intense, reaction for ACE throughout the SFO and suggested that ACE might principally be found in non-neuronal cells. Our localization of immunoreactive ACE to the surfaces of ependymal and glial cells in contact with the CSF and the extracellular spaces near blood vessels in the SFO is especially interesting with regard to the morphological features and transport functions ascribed to these cells. As seen with scanning electron microscopy, the plasma membranes of glial and ependymal cells in contact with extracellular fluids are uniquely characterized by arrays of orthogonally packed particles (assemblies), which are postulated to be sites of transport from the blood and CSF (Brightman et al., 1975; Landis and Reese, 1982). The ependyma and astrocytes in rat brain also show immunocytochemical labeling for certain glycoproteins associated with membranes that possess an electrogenic $\mathrm{Cl}^{-}$pump (Zalc et al., 1984) and have chemically inducible $\mathrm{Na}^{+}$channels (Bowman et al., 1984). The localization of these glycoproteins is consistent with our localization of ACE in non-neuronal cells of the SFO.

Functionally, the ACE located along the plasmalemma of ependyma and astrocytes may be associated with either angiotensin or several other peptides (Defendini et al., 1982). The possible involvement of $\mathrm{ACE}$ in the synthesis of $\mathrm{Ag}$ II from circulating precursors is supported by our demonstration of high levels of AGLI in the extracellular spaces. The glial processes may not only serve in the production of $\mathrm{Ag} \mathrm{II}$, but could isolate extracellular spaces and enclosed neuronal processes, thus slowing down or retarding the diffusion of $\mathrm{Ag}$ II away from perivascular locations or sites of release from nerve terminals. Diffusion barriers for $\mathrm{Ag}$ II would be especially advantageous for the local accumulations of high concentrations of the peptide to act on $\mathrm{Ag}$ II receptors of certain neurons within the SFO (Phillips and Felix, 1976; Simpson et al., 1978; Van Houten et al., 1980).

\section{Luminal surface of capillary endothelium}

The demonstration of peroxidase immunoreactivity for ACE along the luminal surface of capillary endothelial cells is consistent with previous studies in the lung, brain, and other tissues (Brownfield et al., 1982; Ryan et al., 1975). Ultrastructural studies of the localization of ACE immunoreactivity to particulate structures of 80-100 nm diameter on the surface of endothelial cells in the blood vessels of the lung (Caldwell et al., 1976; Ryan et al., 1975) are similar to our observations in the capillaries of the SFO. However, the extent of granulation in our study was highly dependent on the method of tissue preparation, being least noticeable following saponin treatment. Under favorable conditions, other membranes, such as those along the basal and apical portion of ependyma, also showed pronounced granularity. These observations support biochemical evidence that ACE is principally bound to membranes (Soffer, 1976), and they may account for absence of detectable immunoreactivity without lipid extraction to enhance penetration in many regions of the CNS.

\section{Axon terminals and dendrites of neurons}

This study presents the first ultrastructural localization of immunoreactivity for ACE within neurons of the CNS. Previous light-microscopic immunocytochemical (Defendini et al., 1982) and lesion studies using ibotenic acid (Fuxe et al., 1980) suggested that in the basal ganglia $\mathrm{ACE}$ was at least partially located in neurons. However, the neuronal localization was not confirmed by electron microscopy. Furthermore, the activity of the enzyme in the striatum decreased only $50 \%$ following lesions with ibotenic acid, suggesting that ACE was probably also found in afferent terminals or in glia (Fuxe et al., 1980).

Our localization of immunoreactive ACE along the plasmalemma and saccules of smooth reticulum and particles in preterminal axons and dendrites is consistent with the glycoprotein characteristics of the enzyme (Hartley and Soffer, 1978). On the basis of light-microscopic immunocytochemistry, Defendini et al. (1982) previously suggested that ACE might be associated with the plasmalemma of dendrites in the globus pallidus. A similar cellular localization along the axons and dendrites has been reported for glycoconjugates using concanavalin A (Con A) peroxidase conjugates and histochemistry (Hart and Wood, 1984). The smooth membrane profiles near the plasmalemma in axon terminals labeled with Con $A$ are believed to participate in the fast transport of cell-surface glycoconjugates, as well as lectins, introduced exogenously into the cell (Hart and Wood, 1984; LaVail et al., 1983). We also detected peroxidase labeling for ACE within the cisternae of a few large membrane-bound vesicles that appeared to be formed by an infolding of the presynaptic plasma membrane, as was also described for Con $\mathrm{A}$ in axon terminals by Hart and Wood (1984). Our findings differ from the studies of Con $A$, however, in that immunoreactivity was not detected in the saccules of rough endoplasmic reticulum or Golgi apparatus, which are believed to be involved in the production and packaging of glycoproteins destined for the cell surface (Rothman, 1981). Failure to detect ACE within these organelles may reflect lack of accessibility to antigenic sites or conformational changes such that the antiserum recognized the ACE only after more complete assembly within the distal membranes.

In this study, ACE immunoreactivity was also seen around both small and large synaptic vesicles. At least some of the final processing of the immunoreactive enzyme could occur in the synaptosomes, which are known to have glycosyltransferase activity (Preti et al., 1980). Furthermore, ACE has been detected in synaptosomes in ultracentrifugation studies (Paul et al., 1983). However, the peroxidase labeling around synaptosomes in our study was not especially intense and it may represent diffusion and subsequent binding of the enzyme during immunocytochemical labeling.

\section{Cross-reactivity and ultrastructural localization of Ag II antisera}

\section{Cross-reactivity}

Our results demonstrate that the $\mathrm{CE}$ and $\mathrm{DE}$ antisera produced against $\mathrm{Ag}$ II conjugates cross-reacted principally with $\mathrm{Ag}$ II and III. These observations closely correspond to the results obtained by radioimmunoassay using the same antisera (Brownfield et al., 1982). Even though the antisera cross-react with both $\mathrm{Ag}$ II and III, the AGLI detected in the present and earlier studies probably represents principally $\mathrm{Ag} \mathrm{II}$, since relatively low concentrations of $\mathrm{Ag}$ III are detected in mammalian brain (Ganten et al., 1983). However, there remains a possibility that part of the AGLI is attributable to $\mathrm{Ag}$ III or to $\mathrm{Ag} \mathrm{I}, \mathrm{RS}$, or untested peptides. The immunoblots demonstrated that the DE antiserum had greater cross-reaction than the $\mathrm{CE}$ antiserum with 
both $\mathrm{Ag}$ I and RS, which may partially account for the greater ease of detection of the DE antiserum in neuronal perikarya.

\section{Localization in extracellular spaces and axon terminals}

The primary localization of AGLI was in the extracellular spaces near blood vessels and in axon terminals in the central regions of the SFO. The extracellular localization of immunoreactive material is probably unique to circumventricular structures, which have fenestrated capillaries and are thus accessible to circulating hormones (Dellman and Simpson, 1979; Simpson, 1981). The one earlier ultrastructural study of $\mathrm{Ag}$ localization in neurons was within axon terminals in the central nucleus of the amygdala (Paul et al., 1983). The labeled terminals in the amygdala contained numerous large dense-core as well as small clear vesicles and were thus comparable to the first type of axon terminals labeled for $\mathrm{Ag} \mathrm{II}$ in the SFO of the present study. The remaining terminals with AGLI in the SFO of the present study may represent different planes of section through the same type of terminal: Alternatively, the different types of terminals may be attributable to different coexisting transmitters such as $\mathrm{ACh}$ (Hoffman and Phillips, 1977) or to the intrinsic versus extrinsic sources of the labeled axons (Lind et al., 1984).

The present detection of numerous pinocytotic vesicles in portions of dendrites exposed to extracellular spaces and also receiving terminals with AGLI suggests that the same neurons may be receptive to both neuronal and extracellular or circulating $\mathrm{Ag}$ II. A convergence of these 2 pathways was suggested previously by the fact that circulating $\mathrm{Ag}$ II binds most heavily to the central part of the SFO, which also has the greatest density of varicosities with AGLI by light microscopy (Lind et al., 1984; Van Houten et al., 1980).

\section{Localization of $A G L I$ in perikarya and dendrites}

This study is the first to demonstrate the ultrastructural localization of AGLI in perikarya and dendrites in the SFO. Earlier light-microscopic studies using the DE antiserum to Ag showed AGLI in a much larger number of perikarya, which were distributed around the peripheral boundaries of the SFO (Lind et al., 1984). Although the number of cells in the present study was considerably smaller, their location was essentially the same as that described by Lind et al. (1984). The smaller number of labeled cells in our study probably reflects the absence of colchicine treatment, as well as the use of Sprague-Dawley rather than Brattleboro rats.

Our demonstration that neurons with AGLI receive both somatic and dendritic synapses from unlabeled axon terminals indicates that their output is at least partially regulated by intrinsic neurons or afferents containing transmitters other than Ag. A number of afferents to the SFO have been identified in the preoptic and hypothalamic areas (Lind et al., 1984), and they may provide important neuronal regulation for the AGlabeled neurons in the SFO.

\section{Coexistence of immunolabels for $A C E$ and $A g I I$}

The dual localization of AGLI and ACE in neuronal processes in the SFO provides the first electron-microscopic evidence that at least 2 components of the renin-angiotensin system are present within the same central neurons. Earlier light-microscopic studies suggested that both $\mathrm{Ag}$ I and $\mathrm{Ag}$ II were contained within the same neurons in the paraventricular nuclei of the hypothalamus (Healey and Printz, 1984). These immunocytochemical findings provide additional evidence in favor of the hypothesis that neurons synthesize $\mathrm{Ag}$ II, as suggested by several other noncytological lines of evidence, including (1) the detection of renin in synaptosomes from the brains of nephrectomized rats (Paul et al., 1983); (2) the incorporation of tritiated amino acids into $\mathrm{Ag}$ II in single cells (Raizada et al., 1983); and (3) the detection of renin, $\mathrm{Ag}$, and ACE in neuroblastoma cells (Okamura et al., 1981). The relatively small number of processes containing both AGLI and ACE does not necessarily minimize the functional implications of the coexisting antigens. A number of technical reasons may account for the detection of many more peroxidase-labeled processes with ACE than with the gold markcr for Ag. Most importantly, the $10 \mathrm{~nm}$ gold particles have extremely low penetration into the tissue. Thus, only those processes near the surface have levels of immunoreactivity that are clearly distinguishable from the background labeling.

The present detection of ACE immunoreactivity along the plasmalemma and associatcd vesicles and saccules of smooth membrane and comparable local aggregates of AGLI along the plasmalemma in certain axon terminals and dendrites suggests that the active cleavage of $\mathrm{Ag}$ I or other substrates may occur primarily near the neuronal surface. ACE may thus use either locally synthesized products or substrates removed from the extracellular fluid by receptor-mediated uptake (Cantin et al., 1984) or bulk endocytosis (Broadwell and Brightman, 1976).

\section{References}

Bowman, C. L., H. K. Kimelberg, M. V. Frangakis, Y. Berwald-Netter, and C. Edwards (1984) Astrocytes in primary culture have chemically activated sodium channels. J. Neurosci. 4: 1527-1534.

Brightman, M. W., L. Prescott, and T. Reese (1975) Intercellular junctions of special ependyma. In Brain Endocrine Interaction: Vol. 2, Ventricular System, K. M. Knigge, D. E. Scott, M. Kobayahi, and S. Ishii, eds., pp. 146-165, Karger, Basel

Broadwell, R. D., and M. W. Brightman (1976) Entry of peroxidase into neurons of the central and peripheral nervous system from the extracerebral and cerebral blood. J. Comp. Neurol. 166: 257-284.

Brownfield, M. S., L. A. Reid, D. Ganten, and W. F. Ganong (1982) Differential distribution of immunoreactive angiotensin and angiotensin converting enzyme in rat brain. Neuroscience 7: 1759-1769.

Caldwell, P. R. B., B. C. Seegal, K. C. Wsu, M. Das, and R. L. Soffer (1976) Angiotensin converting enzymes: Vascular endothelial localization. Science 191: 1050-1051.

Cantin, M., J. Gutkowska, J. Lacasse, M. Ballak, S. Ledoux, T. Inagami, J. Beuzeron, and J. Genest (1984) Ultrastructural immunocytochemical localization of renin and angiotensin II in the juxtaglomerular cells of the ischemic kidney in experimental renal hypertension. Am. J. Phys. 115: 212-224.

Das, M., and R. L. Soffer (1976) Pulmonary angiotensin-converting enzyme antienzyme. Biochemistry 15: 5088-5094.

Defendini, R., E. A. Zimmerman, J. A. Weare, F. Alhenc-Gelas, and E. G. Erdos (1982) Hydrolysis of enkephalins by human converting enzyme and localization of the enzyme in neuronal components of the brain. In Regulatory Peptides from Molecular Biology to Function, E. Costa and M. Trabucchi, eds., pp. 271-280, Raven, New York.

Dellman, H. D., and J. B. Simpson (1979) The subfornical organ. Int. Rev. Cytol. 58: 333-421.

De Mey, J., A. M. Lambert, A. S. Bajer, M. Moeremans, and M. Brabander (1982) Visualization of microtubules in interphase and mitotic plant cells of haematus endosperm with the immuno-gold staining method. Proc. Natl. Acad. Sci. USA 79: 1898-1902.

Fuxe, K., D. Ganten, Chr. Köhler, B. Schüll, and G. Speck (1980) Converting enzyme and renin in the striatum of rat. Acta Physiol. Scand. 110: 321-323.

Ganten, D., K. Hermann, C. Bayer, T. H. Unger, and R. E. Lang (1983) Angiotensin synthesis in the brain and increased turnover in hypertensive rats. Science 221: 869-871.

Hart, C. E., and J. G. Wood (1984) Endoglycosidase H digestion of neuronal intracellular lectin-binding sites. J. Neurosci. 4: 1589-1597.

Hartley, J. L., and R. L. Soffer (1978) On the oligosaccharide moiety of angiotensin converting enzyme. Biochem. Biophys. Res. Commun. 83: $1545-1552$.

Healy, D. P., and M. P. Printz (1984) Distribution of immunoreactive angiotensin $I$, angiotensinogen, and renin in the central nervous system of intact and nephrectomized rats. Hypertension (Suppl. I) 6: I-130-I-136. 
Hoffman, W. E., and M. I. Phillips (1977) Independent receptors for pressor and drinking responses to central injections of angiotensin II and carbacol. Brain Res. 124: 305-315.

Landis, D. M. D., and T. S. Reese (1982) Regional organization of astrocytic membranes in cerebellar cortex. Neuroscience 7:934-950.

Larsson, L. I. (1981) A novel immunocytochemical model system for specificity sensitivity screening of antisera against multiple antigens. J. Histochem. Cytochem. 29: 408-410.

LaVail, T. N., I. K. Sugino, and D. M. McDonald (1983) Localization of axonally transported ${ }^{125} \mathrm{I}$-wheat germ agglutinin beneath the plasma membrane of chick retinal ganglion cells. J. Cell Biol. 96: 373-381.

Lind, R. W., L. W. Swanson, and D. Ganten (1984) Angiotensin II immunoreactivity in neural afferents and efferents. Brain Res. 321: 209-215.

Lind, R. W., L. E. Ohman, M. B. Lansing, and A. K. Johnson (1983) Transection of subfornical organ neural connections diminishes the pressor response to intravenous infused angiotensin II. Brain Res. 275: 361-364.

Okamura, T., D. L. Clemens, and T. Inagami (1981) Renin, angiotensins, and angiotensin-converting enzyme in neuroblastoma cells: Evidence for intracellular formation of angiotensin. Proc. Natl. Acad. Sci. USA 78: 6940-6943.

Paul, M., K. Hermann, M. Printz, R. E. Lang, T. H. Unger, and D. Ganten (1983) The brain angiotensin system: Subcellular localization and interferences with converting enzyme inhibitors. J. Hypertension (Suppl. 1) 1: 9-15.

Phillips, M. I., and D. Felix (1976) Specific angiotensin II receptive neurons in the cat subfornical organ. Brain Res. 109: 531-540.

Pickel, V. M. (1981) Immunocytochemical methods. In Neuroanatomical Tract Tracing Methods, L. Heimer and J. T. Robards, eds., pp. 483-509, Plenum, New York.

Preti, A., A. Fiorilli, A. Lombardo, L. Caimi, and G. Tettamant (1980)
Occurrence of sialyltransferase activity in synaptosomal membranes prepared from calf brain cortex. J. Neurochem. 35: 28 1-296.

Raizada, M. K., M. I. Phillips, and J. S. Gerndt (1983) Primary cultures from fetal rat brain incorporate $\left({ }^{3} \mathrm{H}\right)$-isoleucine and ${ }^{3} \mathrm{H}$-valine into immunoprecipitable angiotensin II. Neuroendocrinology 36: 64-67.

Rix, E., K. Fuxe, D. Ganten, T. Hökfelt, V. Locatelli, K. Poulsen, G. Stock, and R. Taugner (1981) Renin: Immunocytochemistry in the rat and mouse brain. In Central Nervous System Mechanism in Hypertension, J. P. Buckley and Carlos M. Ferrario, eds., pp. 301-309, Raven, New York.

Rothman, J. E. (1981) The Golgi apparatus: Two organelles in tandem. Science 213: 1212-1219.

Ryan, J. W., U. S. Ryan, D. R. Schultz, C. Whitaker, A. Chung, and R. E. Dorer (1975) Subcellular localization of pulmonary angiotensin-converting enzyme (Kinase II). Biochem. J. 146: 497-499.

Simpson, J. B. (1981) The circumventricular organs and the central actions of angiotensin. Neuroendocrinology 32: 248-256.

Simpson, J. B., A. N. Epstein, and J. S. Camardo (1978) Localization of receptors for the dipsogenic action of angiotensin II in the subfornical organ of the rat. J. Comp. Physiol. Psychol. 92: 581-601.

Soffer, R. L. (1976) Angiotensin-converting enzyme and the regulation of vasoactive peptides. Annu. Rev. Biochem. 45: 73-94.

Sternberger, L. A. (1974) Immunocytochemistry, Prentice-Hall, Englewood Cliffs, NJ.

Van Houten, M., E. L. Schiffrin, J. F. E. Mann, B. I. Posner, and R. Boucher (1980) Radioautographic localizations of specific binding sites for blood-borne angiotensin II in rat brain. Brain Res. 186: 480485.

Zalc, B., A. Collet, M. Monge, M. P. Ollier-Hartmann, C. Jacques, L. Hartman, and N. A. Baumann (1984) Tamm-Horsfall protein, a kidney marker expressed on brain sulfogalactosylcermide-positive astroglial structures. Brain Res. 291: 182-187. 